\title{
ADAPTIVE PDE OBSERVER FOR BATTERY SOC/SOH ESTIMATION
}

\author{
Scott J. Moura \\ Miroslav Krstic \\ Cymer Center for Control Systems and Dynamics \\ Mechanical and Aerospace Engineering \\ University of California, San Diego \\ La Jolla, California 92093 \\ smoura@ucsd.edu,krstic@ucsd.edu
}

\author{
Nalin A. Chaturvedi \\ Research and Technology Center \\ Robert Bosch LLC \\ Palo Alto, California 94304 \\ nalin.chaturvedi@us.bosch.com
}

\begin{abstract}
This paper develops an adaptive PDE observer for battery state-of-charge (SOC) and state-of-health ( $\mathrm{SOH}$ ) estimation. Realtime state and parameter information enables operation near physical limits without compromising durability, thereby unlocking the full potential of battery energy storage. SOC/SOH estimation is technically challenging because battery dynamics are governed by electrochemical principles, mathematically modeled by partial differential equations (PDEs). Simultaneous state and parameter estimation is extremely challenging in PDE models. Consequently, several new theoretical ideas are developed, integrated together, and tested. These include a backstepping PDE state estimator, a Padé-based parameter identifier, nonlinear parameter sensitivity analysis, and adaptive inversion of nonlinear output functions. The end result is the first combined $\mathrm{SOC} / \mathrm{SOH}$ battery estimation algorithm that identifies physical system variables via an electrochemical model, from measurements of voltage and current only.
\end{abstract}

\section{NOMENCLATURE}

A Cell cross sectional area $\left[\mathrm{m}^{2}\right]$

$a^{j} \quad$ Specific interfacial surface area $\left[\mathrm{m}^{2} / \mathrm{m}^{3}\right]$

$c_{e}^{0} \quad$ Li concentration in electrolyte phase $\left[\mathrm{mol} / \mathrm{m}^{3}\right]$

$c_{s}^{j} \quad$ Li concentration in solid phase $\left[\mathrm{mol} / \mathrm{m}^{3}\right]$

$c_{s s}^{j} \quad$ Li concentration at particle surface $\left[\mathrm{mol} / \mathrm{m}^{3}\right]$

$c_{s, \max }^{j}$ Max Li concentration in solid phase $\left[\mathrm{mol} / \mathrm{m}^{3}\right]$

$D_{s}^{j} \quad$ Diffusion coefficent in solid phase $\left[\mathrm{m}^{2} / \mathrm{sec}^{3}\right]$

$F \quad$ Faraday's constant [C/mol]

$I$ Input current [A]

$i_{0}^{j} \quad$ Exchange current density [V] $j \quad$ Positive (+) or negative (-) electrode

$k^{j} \quad$ Reaction rate $\left[\mathrm{A} \cdot \mathrm{mol}^{1.5} / \mathrm{m}^{5.5}\right.$ ]

$L^{j} \quad$ Electrode thickness [m]

$n_{L i} \quad$ Total number of Li ions [mol]

$q$ Boundary input coefficient parameter

$R \quad$ Universal gas constant [J/mol-K]

$R_{f} \quad$ Lumped current collector resistance $[\Omega]$

$R_{S}^{j} \quad$ Particle radius [m]

$r$ Radial coordinate $[\mathrm{m}]$ or $[\mathrm{m} / \mathrm{m}]$

$T$ Cell temperature $[\mathrm{K}]$

$t$ Time [sec] or [sec/sec]

$U^{j} \quad$ Equilibrium potential [V]

$V \quad$ Output voltage [V]

$\alpha^{j} \quad$ Anodic/cathodic transfer coefficient

$\varepsilon$ Diffusion parameter

$\varepsilon_{s}^{j} \quad$ Volume fraction of solid phase

\section{INTRODUCTION}

This paper investigates an adaptive PDE observer for combined state-of-charge (SOC) and state-of-health ( $\mathrm{SOH}$ ) estimation in batteries, using an electrochemical model.

Accurate battery SOC estimation algorithms are currently of extreme importance due to their applications in electrified transportation and energy storage systems for renewable sources. The relevancy of this topic is further underscored by the 27.2 billion USD federal government investment in energy efficiency and renewable energy research, including advanced batteries, under the American Recovery and Reinvestment Act (ARRA) of 2009. As such, battery management systems within these advanced transportation and energy infrastructures must have accurate knowl- 
edge of internal battery energy levels [1]. Such knowledge enables them to efficiently route energy while satisfying power demands and device-level operating constraints [2].

Monitoring battery SOC and SOH is particularly challenging for several technical reasons. First, directly measuring Li concentration or physical examination of cell components is impractical outside specialized laboratory environments $[3,4]$. Second, the dynamics are governed by partial differential algebraic equations derived from electrochemical principles [5]. The only measurable quantities (voltage and current) are related to the states through boundary values. Finally, the model's parameters vary widely with electrode chemistry, packaging, and time. In this paper we directly address these technical challenges. Namely, we design an adaptive observer using a reduced-form PDE model based upon electrochemical principles. As such, the algorithm estimates physical variables directly related $\mathrm{SOC}$ and $\mathrm{SOH}$, a first to the authors' knowledge.

Over the past decade research on battery $\mathrm{SOC} / \mathrm{SOH}$ estimation has experienced considerable growth. One may divide this research by the battery models each algorithm employs.

The first category considers estimators based upon equivalent circuit models (ECMs). These models use circuit elements to mimic the phenomenological behavior of batteries. For example, the seminal work by Plett [6] applies an extended Kalman filter to simultaneously identify the states and parameters of an ECM. Verbrugge and his co-workers used ECMs with combined coulumb-counting and voltage inversion techniques in [7] and adaptive parameter identification algorithms in [8]. More recently, a linear parameter varying approach was designed in [9]. The key advantage of ECMs is their simplicity. However, they often require extensive parameterization for accurate predictions. This often produces models with non-physical parameters, whose complexity becomes comparable to electrochemical models.

The second category considers electrochemical models, which account for the diffusion, intercalation, and electrical dynamics. Although these models can accurately predict internal state variables, their mathematical structure is generally too complex for controller/observer design. Therefore, these approaches combine model reduction and estimation techniques. Some of the first studies within this category use a "single particle model" (SPM) of electrochemical battery dynamics in combination with an extended Kalman filter [10,11]. A separate research group employed residue grouping for model reduction and linear Kalman filters for observers [12]. The authors of [13] apply simplifications to the electrolyte and solid phase concentration dynamics to perform SOC estimation. To date, however, simultaneous SOC and $\mathrm{SOH}$ estimation using electrochemical models remains an open question.

In this paper we extend the aforementioned research by designing an electrochemical model based adaptive observer for simultaneous SOC/SOH estimation. Several novel theoretical ideas are developed, integrated, and tested. These include a PDE backstepping state estimator, Padé-based PDE parameter identifier, nonlinear identifiability analysis of the output equation, and adaptive output function inversion. The final result is an adaptive observer for simultaneous SOC/SOH estimation which identifies physical battery system variables, from current and voltage measurements only.

The paper is organized as follows: Section 2 describes the electrochemical-based single particle model. Sections 3-6 describe the subsystems of the adaptive observer, including the state estimator, PDE parameter identifier, output function parameter identifier, and adaptive output function inversion. Sections 7 and 8 respectively provide simulation results and a summary of the key contributions.

\section{ELECTROCHEMICAL CELL MODEL \& ANALYSIS}

The single particle model (SPM) was first applied to lithium battery systems in [14] and is the model we utilize in this work. The key idea is that the solid phase of each electrode can be idealized as a single spherical particle. This model results if one assumes the electrolyte Li concentration is constant in space and time. This assumption works well for small currents or electrolytes with large electronic conductivities. However, it induces errors at large C-rates. Moreover, we assume constant temperature. Figure 1 provides a schematic of the SPM concept. Mathematically, the model consists of two diffusion PDEs governing each electrode's concentration dynamics, where input current enters as a Neumann boundary condition. Output voltage is given by a nonlinear function of the state values at the boundary and the input current.

Although this model captures less dynamic behavior than other electrochemical-based estimation models [14], its mathematical structure is amenable to adaptive observer design.

\subsection{Single Particle Model}

Diffusion in each electrode is governed by Fick's law in spherical coordinates:

$$
\begin{aligned}
& \frac{\partial c_{s}^{-}}{\partial t}(r, t)=D_{s}^{-}\left[\frac{2}{r} \frac{\partial c_{s}^{-}}{\partial r}(r, t)+\frac{\partial^{2} c_{s}^{-}}{\partial r^{2}}(r, t)\right] \\
& \frac{\partial c_{s}^{+}}{\partial t}(r, t)=D_{s}^{+}\left[\frac{2}{r} \frac{\partial c_{s}^{+}}{\partial r}(r, t)+\frac{\partial^{2} c_{s}^{+}}{\partial r^{2}}(r, t)\right]
\end{aligned}
$$

with Neumann boundary conditions:

$$
\begin{aligned}
& \frac{\partial c_{s}^{-}}{\partial r}(0, t)=0, \quad \frac{\partial c_{s}^{-}}{\partial r}\left(R_{s}^{-}, t\right)=-\frac{I(t)}{D_{s}^{-} F a^{-} A L^{-}} \\
& \frac{\partial c_{s}^{+}}{\partial r}(0, t)=0, \quad \frac{\partial c_{s}^{+}}{\partial r}\left(R_{s}^{+}, t\right)=\frac{I(t)}{D_{s}^{+} F a^{+} A L^{+}}
\end{aligned}
$$

The Neumann boundary conditions at $r=R_{s}^{+}$and $r=R_{s}^{-}$signify that the flux entering the electrode is proportional to the input current $I(t)$. The Neumann boundary conditions at $r=0$ are 


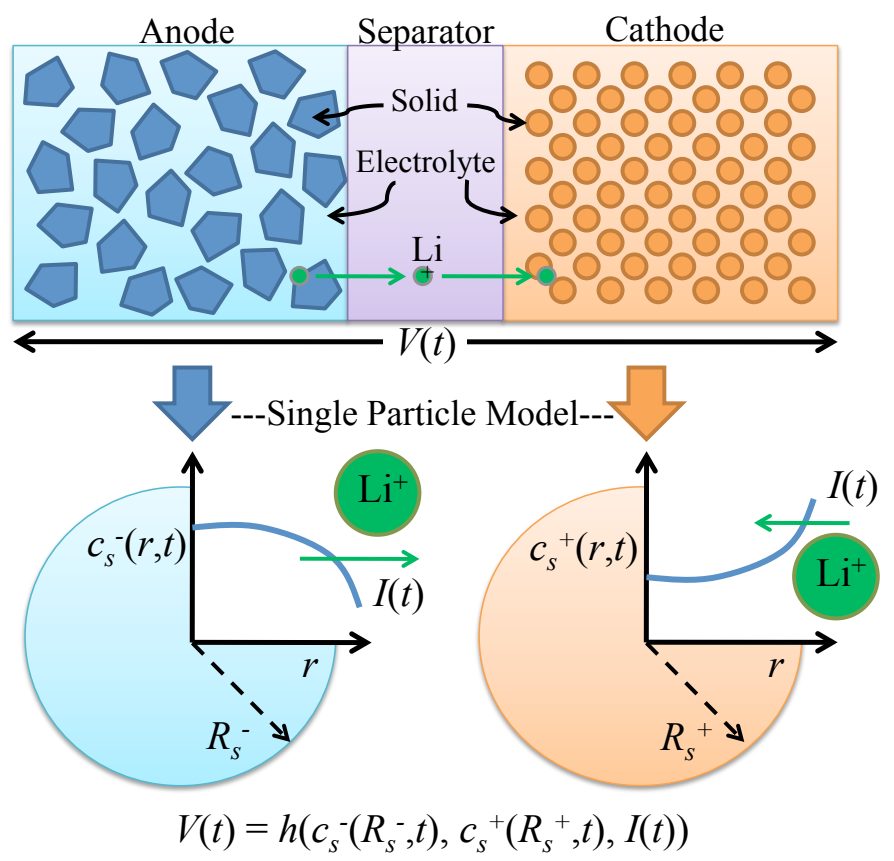

Figure 1. EACH ELECTRODE IS IDEALIZED AS A SINGLE POROUS SPHERICAL PARTICLE. DURING CHARGING/DISCHARGING, LI INTERCALATES INTO/OUT OF THESE SPHERICAL PARTICLES.

required for well-posedness. Note that the states for the two PDEs are dynamically uncoupled, although they have proportional boundary inputs.

The measured terminal voltage output is governed by a combination of electric overpotential, electrode thermodynamics, and Butler-Volmer kinetics. The end result is:

$$
\begin{aligned}
V(t)= & \frac{R T}{\alpha F} \sinh ^{-1}\left(\frac{I(t)}{2 a^{+} A L^{+} i_{0}^{+}\left(c_{s s}^{+}(t)\right)}\right) \\
& -\frac{R T}{\alpha F} \sinh ^{-1}\left(\frac{I(t)}{2 a^{-} A L^{-} i_{0}^{-}\left(c_{s s}^{-}(t)\right)}\right) \\
& +U^{+}\left(c_{s s}^{+}(t)\right)-U^{-}\left(c_{s s}^{-}(t)\right)-R_{f} I(t)
\end{aligned}
$$

where the exchange current density $i_{0}^{j}$ and solid-electrolyte surface concentration $c_{s s}^{j}$ are, respectively:

$$
\begin{aligned}
i_{0}^{j}\left(c_{s s}^{j}\right) & =k^{j} \sqrt{c_{e}^{0} c_{s s}^{j}(t)\left(c_{s, \max }^{j}-c_{s s}^{j}(t)\right)} \\
c_{s s}^{j}(t) & =c_{s}^{j}\left(R_{s}^{j}, t\right), \quad \mathrm{j} \in\{+,-\}
\end{aligned}
$$

The functions $U^{+}(\cdot)$ and $U^{-}(\cdot)$ are the equilibrium potentials of each electrode material, given the surface concentration. Mathematically, these are strictly monotonically decreasing functions of their input. This fact implies that the inverse of its derivative is always finite, a property which we require later. Further details on the electrochemical principles used to derive these equations can be found in $[1,5]$.
This model contains the property that the total number of lithium ions is conserved [13]. Mathematically, $\frac{d}{d t}\left(n_{L i}\right)=0$

$$
\begin{aligned}
n_{L i}= & \frac{\varepsilon_{s}^{+} L^{+} A}{\frac{4}{3} \pi\left(R_{s}^{+}\right)^{3}} \int_{0}^{R_{s}^{+}} 4 \pi r^{2} c_{s}^{+}(r, t) d r \\
& +\frac{\varepsilon_{s}^{-} L^{-} A}{\frac{4}{3} \pi\left(R_{s}^{-}\right)^{3}} \int_{0}^{R_{s}^{-}} 4 \pi r^{2} c_{s}^{-}(r, t) d r
\end{aligned}
$$

This property will become important, as it relates the total concentration of lithium in the cathode and anode. We leverage this fact to perform model reduction in the state estimation problem.

In the following sections we describe each subsystem of the adaptive observer. A block diagram of the composed system is provided in Fig. 2.

\section{STATE ESTIMATION}

\subsection{Observability \& Model Reduction}

For the purpose of observer design we reduce the SPM by approximating the cathode diffusion dynamics (2) by its equilibrium. This step is mathematically motivated by the fact that the SPM states are weakly observable from voltage measurements, as has been previously noted in the literature [11]. It turns out that approximating the cathode dynamics as instantaneous produces a reduced system whose states are locally strongly observable. Moreover, physical motivation exists for this reduction when diffusion dynamics are significantly faster in the cathode than the anode, a common characteristic of certain anode/cathode combinations. We discuss these points in succession.

Lack of observability can be shown using a number of techniques. For example, one may (i) approximate the PDEs by ODEs using the finite difference method, producing a tri-diagonal matrix $A$, (ii) linearize the output equation about the states, producing a matrix $C$, (iii) and compute the rank of the observability matrix for the pair $(A, C)[15]$.

The reduced SPM has a PDE given by (1), boundary conditions given by (3), and output equation:

$$
\begin{aligned}
V(t)= & \frac{R T}{\alpha^{+} F} \sinh ^{-1}\left(\frac{I(t)}{2 a^{+} A L^{+} i_{0}^{+}\left(\alpha c_{s s}^{-}(t)+\beta\right)}\right) \\
& -\frac{R T}{\alpha^{-} F} \sinh ^{-1}\left(\frac{I(t)}{2 a^{-} A L^{-} i_{0}^{-}\left(c_{s s}^{-}(t)\right)}\right) \\
& +U^{+}\left(\alpha c_{s s}^{-}(t)+\beta\right)-U^{-}\left(c_{s s}^{-}(t)\right)-R_{f} I(t)
\end{aligned}
$$

Note that $c_{s s}^{+}(t)$ has been replaced by $\alpha c_{s s}^{-}(t)+\beta$. This is the critical detail of the reduced SPM. The equilibrium of the cathode states (i.e., $\left.c_{s}^{+}(r, t)=c_{s s}^{+}(t)\right)$ can be computed from the conserva- 


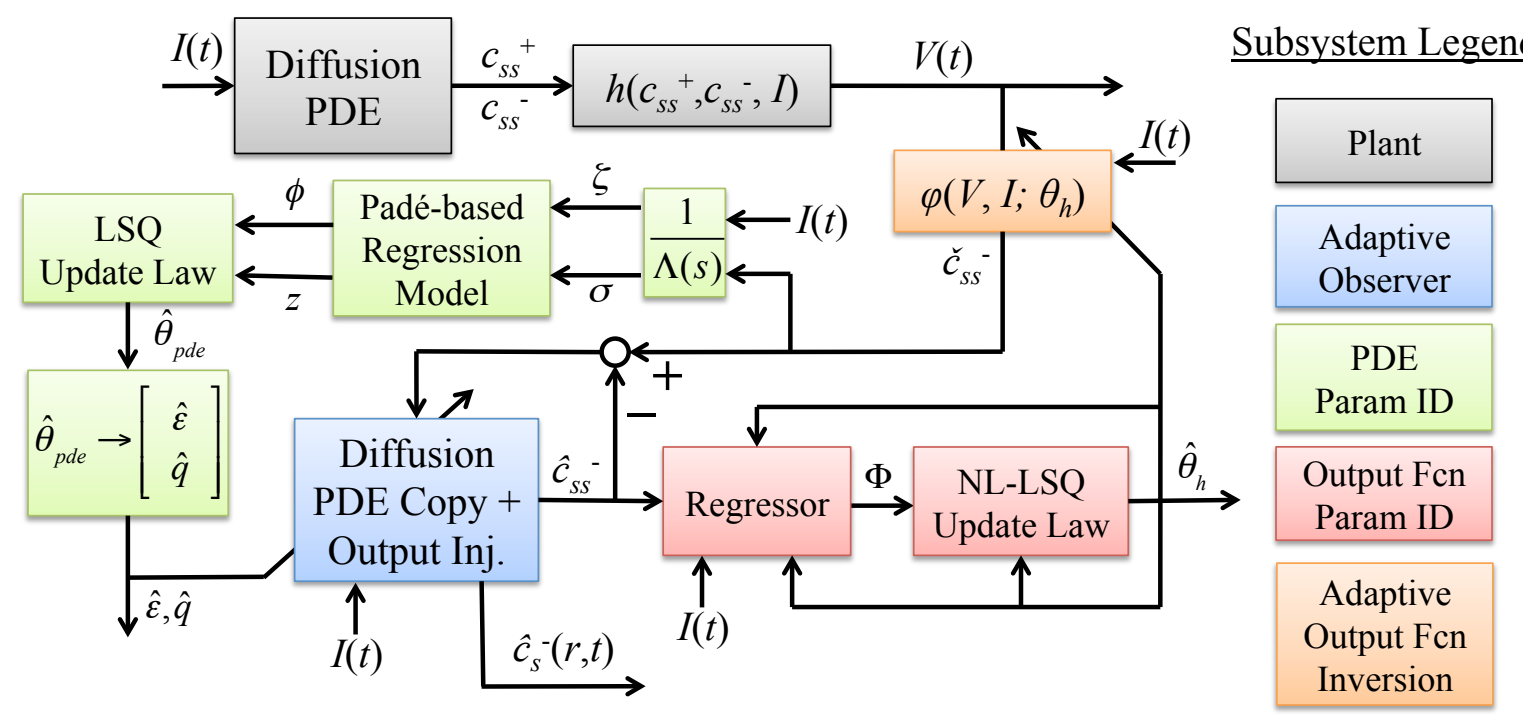

Figure 2. BLOCK DIAGRAM OF ADAPTIVE OBSERVER. IT IS COMPOSED OF THE BACKSTEPPING STATE ESTIMATOR (BLUE), PDE PARAMETER IDENTIFIER (GREEN), OUTPUT FUNCTION PARAMETER IDENTIFIER (RED), AND ADAPTIVE OUTPUT FUNCTION INVERSION (ORANGE).

tion of Li property in (8) to produce the relationship ${ }^{1}$ :

$$
c_{s s}^{+}(t)=\frac{1}{\varepsilon_{s}^{+} L^{+} A}\left[n_{L i}-\varepsilon_{s}^{-} L^{-} A c_{s s}^{-}(t)\right]
$$

where $\alpha=-\frac{\varepsilon_{s}^{-} L^{-}}{\varepsilon_{s}^{+} L^{+}}$and $\beta=\frac{n_{L i}}{\varepsilon_{s}^{+} L^{+} A}$.

One can show this system is locally observable (i.e. in the linear sense) by using the same finite difference and linearization approach described above. Ultimately, we guarantee observability for this reduced SPM by designing the observer gains such that the estimation error dynamics mimic an exponentially stable target system. This is the core concept behind backstepping observer design [16].

Physical motivation sometimes exists for approximating the cathode diffusion dynamics as instantaneous. Significant research efforts on manufacturing and material science techniques for cathode materials has enabled researchers to attain nano-scale particle sizes and faster diffusion rates [17]. The result is characteristic diffusion times (mathematically $R_{s}^{2} / D_{s}$ ) which are often orders of magnitude less in the cathode than the anode. Parallel studies have been performed on the anode side (see e.g. [18]), however they are less prevalent. Hence, approximating cathode diffusion by its equilibrium is a reasonable approximation for certain cathode/anode combinations. This insight was also observed through a previous parameter identification study on commercially available

\footnotetext{
${ }^{1}$ To be technically correct, the cathode concentration should depend on the anode concentration summed over the spherical volume: $c_{S S}^{+}(t)=$ $\frac{1}{\varepsilon_{s}^{+} L^{+} A}\left[n_{L i}-\frac{3 \varepsilon_{s}^{-} L^{-} A}{4 \pi R_{s}^{-3}} \int_{0}^{R_{s}^{-}} 4 \pi r^{2} c_{s}^{-}(r, t) d r\right]$. However, this results in a nonlinear output equation which depends on the in-domain states, as well as the boundary state. This would create additional complexity to the backstepping approach we employ in this paper.
}

$\mathrm{LiFePO}_{4}$ cells with doped nano-scale cathode materials [19]. For other cells, the requisite diffusive time scale separation property may not exist.

The SPM comprises linear dynamics and a nonlinear output function. In general an output injection-based estimator would be nonlinear for this class of systems. However, we design a linear estimator in this paper by injecting the boundary state error. This idea requires us to calculate the boundary state from the measured voltage, demonstrated visually by the block diagram in Fig. 2 . In [20] we show the output function (9) is invertible with respect to the boundary state $c_{s s}^{-}$, uniformly in the input current $I(t)$.

\subsection{Normalization and State Transformation}

Next we perform normalization and state transformation to simplify the mathematical structure of the observer. First scale the radial $r$ and time $t$ coordinates as follows:

$$
\bar{r}=\frac{r}{R_{s}^{-}} \quad \bar{t}=\frac{D_{s}^{-}}{\left(R_{s}^{-}\right)^{2}} t
$$

Henceforth we will drop the bars over the space and time coordinates to simplify notation. Next we perform a state transformation to eliminate the first spatial derivative in the spherical diffusion equation (1). Namely, let

$$
c(r, t)=r c_{s}^{-}(r, t)
$$


This normalization and state transformation produces the following PDE with Dirichlet and Robin boundary conditions:

$$
\begin{aligned}
\frac{\partial c}{\partial t}(r, t) & =\varepsilon \frac{\partial^{2} c}{\partial r^{2}}(r, t) \\
c(0, t) & =0 \\
\frac{\partial c}{\partial r}(1, t)-c(1, t) & =-q \rho I(t)
\end{aligned}
$$

and nonlinear output map given by (9) where $c_{s s}^{+}=\alpha c(1, t)+\beta$ (see (10)) and $c_{s s}^{-}=c(1, t)$. The parameter $\rho=R_{s}^{-} /\left(D_{s}^{-} F a^{-} A L^{-}\right)$ groups together known parameters. The parameters $\varepsilon$ and $q$ are nominally equal to one. Respectively, they represent uncertainty in the diffusion and boundary input coefficients, which we identify in Section 4.

\subsection{Backstepping PDE State Estimator}

In previous work [20], we designed a backstepping PDE state estimator for the SPM. The state estimator structure consists of a copy of the plant (13)-(15) plus boundary state error injection, as follows:

$$
\begin{aligned}
\frac{\partial \hat{c}}{\partial t}(r, t) & =\varepsilon \frac{\partial^{2} \hat{c}}{\partial r^{2}}(r, t)+p_{1}(r) \tilde{c}(1, t) \\
\hat{c}(0, t) & =0 \\
\frac{\partial \hat{c}}{\partial r}(1, t)-\hat{c}(1, t) & =-q \rho I(t)+p_{10} \tilde{c}(1, t)
\end{aligned}
$$

where the boundary state error is given by:

$$
\tilde{c}(1, t)=\varphi(V(t), I(t))-\hat{c}(1, t)
$$

and the function estimation gains are given by:

$$
\begin{aligned}
p_{1}(r) & =\frac{-\lambda r}{2 \varepsilon z}\left[I_{1}(z)-\frac{2 \lambda}{\varepsilon z} I_{2}(z)\right] \\
\text { where } z & =\sqrt{\frac{\lambda}{\varepsilon}\left(r^{2}-1\right)} \\
p_{10} & =\frac{1}{2}\left(3-\frac{\lambda}{\varepsilon}\right)
\end{aligned}
$$

and $I_{1}(z)$ and $I_{2}(z)$ are, respectively, the first and second order modified Bessel functions of the first kind. Please consult [20] for the stability proof.

Note that the estimator is linear in the state because we use the boundary state for error injection. The plant boundary state is computed by inverting the nonlinear output mapping w.r.t. the boundary state, given a current input (i.e. $\varphi(V(t), I(t)))$. The output function inversion is discussed in detail in Section 6.
Also note the parameters $\varepsilon$ in (16), (20)-(22) and $q$ in (18). In the subsequent section we design an identifier for these parameters. We form an adaptive observer by replacing these parameters with their estimates, via the certainty equivalence principle [21].

\section{PDE PARAMETER IDENTIFICATION}

Next we design an identification algorithm for the diffusion and boundary input coefficients in (13) and (15), respectively. Identification of the diffusion coefficient $\varepsilon$ from boundary measurements is a significant fundamental challenge [22], for the following reason. In finite-dimensional state-space systems we typically write the system in observable canonical form. This structure enables one to uniquely identify state-space parameters from input-output data. In our problem we require a parametric model where the diffusion coefficient multiplies measured data only. There is no clear way to do this for PDEs. This motivates our new contribution: utilizing a reduced-order model (Padé approximation) for the parameter identification.

\subsection{Padé Approximates}

The PDE model (13)-(15) can be written in the frequency domain as a transcendental transfer function:

$$
G(s)=\frac{c_{s s}(s)}{I(s)}=\frac{-q \rho \sinh (\sqrt{s / \varepsilon})}{(\sqrt{s / \varepsilon}) \cosh (\sqrt{s / \varepsilon})-\sinh (\sqrt{s / \varepsilon})}
$$

We now apply Padé approximations of the transcendental transfer function (23). Padé approximants represent a function by a ratio of two power series. The defining characteristic of a Padé approximate is that its Taylor series matches the Taylor series of the function it is approximating. Another useful property of Padé approximates is that they naturally contain poles and zeros. The Padé expansion takes the following form:

$$
G(s)=\lim _{N \rightarrow \infty} \frac{\sum_{k=0}^{N} b_{k} s^{k}}{1+\sum_{k=1}^{N} a_{k} s^{k}}
$$

Figure 3 provides bode plots of $G(s)$ and several Padé approximates. Their analytical expressions are supplied in Table 1. The Padé approximates capture low frequency dynamics well. Accuracy at high frequency increases as the Padé order increases. We low-pass filter the input-output signals such that data is retained where the Padé approximation is sufficiently accurate.

Our immediate goal is to design a parameter identification scheme for the Padé approximation of the original PDE model. 

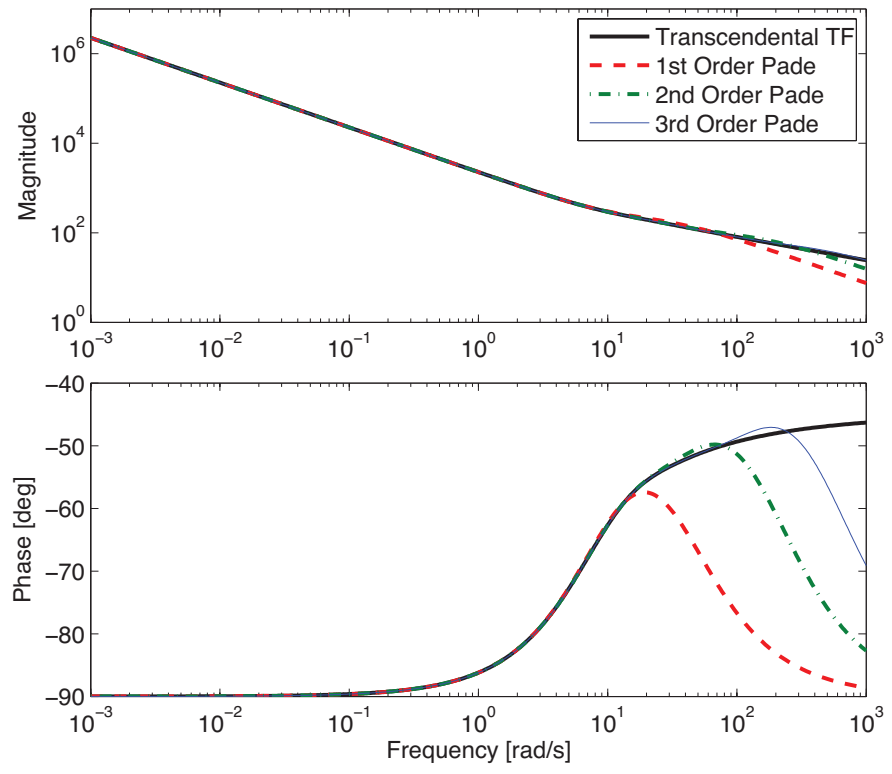

Figure 3. BODE PLOTS OF THE TRANSCENDENTAL TRANSFER FUNCTION (23) AND PADÉ APPROXIMATES IN TABLE 1.

\subsection{Least Squares Identification}

We utilize the first order Padé approximant as the nominal model. Namely,

$$
\frac{C_{s s}(s)}{I(s)} \approx P_{1}(s)=\frac{-3 q \rho \varepsilon-\frac{2}{7} q \rho s}{s+\frac{1}{35 \varepsilon} s^{2}}
$$

Assuming zero initial conditions and applying the inverse Laplace transform produces the following linearly parameterized model:

$$
\frac{1}{35} \ddot{c}_{s s}(t)=-\varepsilon \dot{c}_{s s}(t)-3 \rho q \varepsilon^{2} I(t)-\frac{2}{7} \rho q \varepsilon \dot{I}(t)
$$

Table 1. PADÉ APPROXIMATES OF THE PDE MODEL

(13)-(15)

\begin{tabular}{ll}
\hline \hline Order, $k$ & $P_{k}(s)$ \\
\hline 1 & $\frac{-q \rho\left(\frac{2}{7} s+3 \varepsilon\right)}{s\left(\frac{1}{35 \varepsilon} s+1\right)}$ \\
2 & $\frac{-q \rho\left(\frac{1}{165 \varepsilon} s^{2}+\frac{4}{11} s+3 \varepsilon\right)}{s\left(\frac{1}{3465 \varepsilon^{2}} s^{2}+\frac{3}{55 \varepsilon} s+1\right)}$ \\
3 & $\frac{-q \rho\left(\frac{4}{75075 \varepsilon^{2}} s^{3}+\frac{2}{195 \varepsilon} s^{2}+\frac{2}{5} s+3 \varepsilon\right)}{s\left(\frac{1}{675675 \varepsilon^{3}} s^{3}+\frac{2}{2275 \varepsilon^{2}} s^{2}+\frac{1}{15 \varepsilon} s+1\right)}$ \\
\hline
\end{tabular}

Since the parametric model contains time derivatives of measured signals, we employ filters [21] to avoid differentiation:

$$
\begin{aligned}
& \dot{\sigma}_{1}=\sigma_{2} \\
& \dot{\sigma}_{2}=-\lambda_{0} \sigma_{1}-\lambda_{1} \sigma_{2}+c_{s s} \\
& \dot{\zeta}_{1}=\zeta_{2} \\
& \dot{\zeta}_{2}=-\lambda_{0} \zeta_{1}-\lambda_{1} \zeta_{2}+I
\end{aligned}
$$

where the polynomial $\Lambda(s)=s^{2}+\lambda_{1} s+\lambda_{0}$ is chosen Hurwitz. One can analytically show that selecting the roots of $\Lambda(s)$ results in a trade-off between convergence rate (via level of persistence of excitation) and parameter bias (error induced by Padé approximation). Consequently, the parametric model is:

$$
\frac{1}{35}\left(-\lambda_{0} \sigma_{1}-\lambda_{1} \sigma_{2}+c_{s s}\right)=-3 \rho q \varepsilon^{2} \zeta_{1}-\frac{2}{7} \rho q \varepsilon \zeta_{2}-\varepsilon \sigma_{2}
$$

Let us denote the vector of unknown parameters by:

$$
\theta_{p d e}=\left[q \varepsilon^{2} q \varepsilon \varepsilon\right]^{T}
$$

Then the parametric model can be expressed in matrix form as $z_{p d e}=\theta_{p d e}^{T} \phi$, where

$$
\begin{aligned}
z_{p d e} & =\frac{1}{35}\left(-\lambda_{0} \sigma_{1}-\lambda_{1} \sigma_{2}+c_{s s}\right) \\
\phi & =\left[\begin{array}{lll}
-3 \rho \zeta_{1} & -\frac{2}{7} \rho \zeta_{2} & -\sigma_{2}
\end{array}\right]^{T}
\end{aligned}
$$

Given this linearly parameterized model, we chose a least-squares update law of the form [21]:

$$
\begin{aligned}
\dot{\hat{\theta}}_{p d e} & =P_{p d e} \frac{z_{p d e}-\hat{\theta}_{p d e}^{T} \phi}{m_{p d e}^{2}} \phi \\
\dot{P}_{p d e} & =-P_{p d e} \frac{\phi \phi^{T}}{m_{p d e}^{2}} P_{p d e}, \quad P_{p d e}(0)=P_{p d e 0}=P_{p d e 0}^{T}>0 \\
m_{p d e}^{2} & =1+\gamma_{p d e} \phi^{T} \phi, \quad \gamma_{p d e}>0
\end{aligned}
$$

4.2.1 Managing Overparameterization with the Pseudoinverse An important implementation issue with the proposed Padé approximation approach is overparameterization. That is, the physical parameters must be uniquely determined from the parameter vector $\hat{\theta}_{p d e}$

$$
\hat{\theta}_{p d e}=\left[\begin{array}{c}
\widehat{q \varepsilon^{2}} \\
\widehat{q \varepsilon} \\
\hat{\varepsilon}
\end{array}\right] \longrightarrow\left[\begin{array}{l}
\hat{\varepsilon} \\
\hat{q}
\end{array}\right]=\hat{\theta}_{\varepsilon q}
$$


Coincidently, the particular nonlinear form (products and powers) of the elements in vector $\hat{\theta}_{p d e}$ allows us to write a set of linear equations using a logarithmic nonlinear transformation and properties of the logarithm function:

$$
\left[\begin{array}{ll}
2 & 1 \\
1 & 1 \\
1 & 0
\end{array}\right]\left[\begin{array}{l}
\log \hat{\varepsilon} \\
\log \hat{q}
\end{array}\right]=\left[\begin{array}{c}
\log \left(q \hat{\varepsilon}^{2}\right) \\
\log (\hat{q} \varepsilon) \\
\log (\hat{\varepsilon})
\end{array}\right]
$$

which we re-write into compact notation as

$$
A_{\varepsilon q} \overline{\log }\left(\hat{\theta}_{\varepsilon q}\right)=\overline{\log }\left(\hat{\theta}_{p d e}\right)
$$

where $\overline{\log }(\theta)=\left[\log \left(\theta_{1}\right), \log \left(\theta_{2}\right), \ldots\right]^{T}$. The parameter vector $\hat{\theta}_{\varepsilon q}$ can be uniquely solved from (39) via the Moore-Penrose pseudoinverse. That is:

$$
\overline{\log }\left(\hat{\theta}_{\varepsilon q}\right)=\left(A_{\varepsilon q}^{T} A_{\varepsilon q}\right)^{-1} A_{\varepsilon q}^{T} \overline{\log }\left(\hat{\theta}_{p d e}\right)
$$

This method works well in practice with respect to feeding parameter estimates into the adaptive observer (lower-left-hand block in Fig. 2), since the pseudoinverse ultimately involves computationally efficient matrix algebra.

\section{OUTPUT FUNCTION PARAMETER IDENTIFICATION}

The greatest difficultly in battery estimation arguably stems from the nonlinear relationship between SOC and voltage [9]. We directly address this difficulty by developing an identification algorithm for the uncertain parameters in the nonlinearly parameterized output function (9). First, we analyze parameter identifiability to assess which subset of parameters are uniquely identifiable. Second, we apply nonlinear least squares to this subset.

\subsection{Identifiability}

A necessary first step in nonlinear parameter identification is a parameter sensitivity analysis. We specifically apply the ranking procedure outlined in [23] to assess linear dependence. Consider the output function (9) written in parametric form:

$$
\begin{aligned}
h(t ; \theta)=V(t)= & \frac{R T}{\alpha F} \sinh ^{-1}\left[\frac{\theta_{2} I(t)}{2 \sqrt{c_{s s}^{+}\left(t ; \theta_{1}\right)\left(c_{s, \max }^{+}-c_{s s}^{+}\left(t ; \theta_{1}\right)\right)}}\right] \\
& -\frac{R T}{\alpha F} \sinh ^{-1}\left[\frac{\theta_{3} I(t)}{2 \sqrt{c_{s s}^{-}(t)\left(c_{s, \max }^{-}-c_{s s}^{-}(t)\right)}}\right] \\
& +U^{+}\left(c_{s s}^{+}\left(t ; \theta_{1}\right)\right)-U^{-}\left(c_{s s}^{-}(t)\right)-\theta_{4} I(t)
\end{aligned}
$$

where $c_{s s}^{+}\left(t ; \theta_{1}\right)$ and the parameter vector $\theta$ are

$$
\begin{aligned}
c_{s s}^{+}\left(t ; \theta_{1}\right) & =-\frac{\varepsilon_{s}^{-} L^{-}}{\varepsilon_{s}^{+} L^{+}} c_{s s}^{-}(t)+\frac{\theta_{1}}{\varepsilon_{s}^{+} L^{+} A} \\
\theta & =\left[n_{L i}, \frac{1}{a^{+} A L^{+} k^{+} \sqrt{c_{e}^{0}}}, \frac{1}{a^{-} A L^{-} k^{-} \sqrt{c_{e}^{0}}}, R_{f}\right]^{T}
\end{aligned}
$$

We have selected the elements of $\theta$ because diminishing $n_{L i}$ physically models capacity fade and increasing values for the other parameters capture various forms of internal resistance.

The following sensitivity analysis is performed in discrete time, since the required data is supplied in discrete time. Let $k$ index time such that $t=k \Delta T, k \in 1,2, \ldots, n_{T}$. The sensitivity of the output with respect to variations in the parameter $\theta_{i}$ at time index $k$ is defined as $S_{i, k}=\frac{\partial h(k \Delta T ; \theta)}{\partial \theta_{i}}$. For each parameter $\theta_{i}$, stack the sensitivities at time indeces $k=1,2, \ldots n_{T}$, i.e. $S_{i}=\left[S_{i, 1}, S_{i, 2}, \ldots, S_{i, n_{T}}\right]^{T}$. Denote $S=\left[S_{1}, S_{2}, S_{3}, S_{4}\right]$, such that $S \in \mathbb{R}^{n_{T} \times 4}$. A particular decomposition of $S^{T} S$ reveals useful information about linear dependence between parameters. Let $S^{T} S=D^{T} C D$ where

$$
\begin{aligned}
& D=\left[\begin{array}{cccc}
\left\|S_{1}\right\| & 0 & 0 & 0 \\
0 & \left\|S_{2}\right\| & 0 & 0 \\
0 & 0 & \left\|S_{3}\right\| & 0 \\
0 & 0 & 0 & \left\|S_{4}\right\|
\end{array}\right]
\end{aligned}
$$

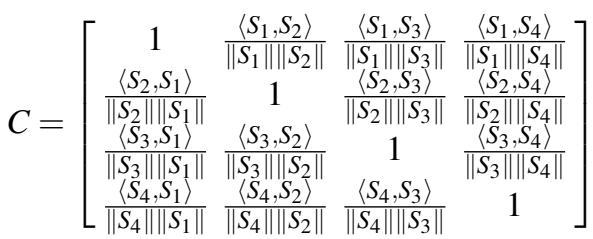

where $\|\cdot\|$ denotes the Euclidian norm and $\langle\cdot, \cdot\rangle$ is the inner product. By the Cauchy Schwarz inequality $-1 \leq \frac{\left\langle S_{i}, S_{j}\right\rangle}{\left\|S_{i}\right\|\left\|S_{j}\right\|} \leq 1$. This has the interpretation that values of $\frac{\left\langle S_{i}, S_{j}\right\rangle}{\left\|S_{i}\right\|\left\|S_{j}\right\|}$ near -1 or 1 imply strong linear dependence between parameters $\theta_{i}$ and $\theta_{j}$, whereas values near zero imply orthogonality.

An example for the matrix $C$ is provided in (45). This example analyzes parameter sensitivity for a UDDS drive cycle data set applied to the SPM battery model.

$$
C=\left[\begin{array}{rrrr}
1 & -0.3000 & 0.2908 & 0.2956 \\
-0.3000 & 1 & -0.9801 & -0.9805 \\
0.2908 & -0.9801 & 1 & 0.9322 \\
0.2956 & -0.9805 & 0.9322 & 1
\end{array}\right]
$$

Note that strong linear dependence exists between $\theta_{2}, \theta_{3}, \theta_{4}$. This property is uniformly true across various drive cycles (e.g. US06, SC04, LA92, naturalistic micro-trips). This means it is difficult to determine how each individual parameter value changes, amongst 
these three parameters. As a result, we identify only two parameters, $n_{L i}$ and $R_{f}$, which capture capacity and power fade, respectively.

\subsection{Nonlinear Least Squares}

Now our immediate goal is to identify the parameter vector $\theta_{h}=\left[\begin{array}{ll}n_{L i} & R_{f}\end{array}\right]^{T}$ via a nonlinear least squares identification algorithm. Define $\tilde{\theta}_{h}=\theta_{h}-\hat{\theta}_{h}$ and write (42) in terms of $\tilde{\theta}_{h}$

$$
\begin{aligned}
V\left(t ; \theta_{h}\right)= & \frac{R T}{\alpha F} \sinh ^{-1}\left[\frac{I(t)}{2 a^{+} A L^{+} i_{0}^{+}\left(c_{s s}^{+}\left(t ; \tilde{\theta}_{h 1}+\hat{\theta}_{h 1}\right)\right)}\right] \\
& -\frac{R T}{\alpha F} \sinh ^{-1}\left[\frac{I(t)}{2 a^{-} A L^{-} i_{0}^{-}\left(c_{s s}^{-}(t)\right)}\right] \\
& +U^{+}\left(c_{s s}^{+}\left(t ; \tilde{\theta}_{h 1}+\hat{\theta}_{h 1}\right)\right)-U^{-}\left(c_{s s}^{-}(t)\right)-\left(\tilde{\theta}_{h 2}+\hat{\theta}_{h 2}\right) I(t)
\end{aligned}
$$

Next we take the Maclaurin series expansion with respect to $\tilde{\theta}_{h}$

$$
\begin{aligned}
V\left(t ; \theta_{h}\right)= & \frac{R T}{\alpha F} \sinh ^{-1}\left[\frac{I(t)}{2 a^{+} A L^{+} i_{0}^{+}\left(c_{s s}^{+}\left(t ; \hat{\theta}_{h 1}\right)\right)}\right] \\
& -\frac{R T}{\alpha F} \sinh ^{-1}\left[\frac{I(t)}{2 a^{-} A L^{-} i_{0}^{-}\left(c_{s s}^{-}(t)\right)}\right] \\
& +U^{+}\left(c_{s s}^{+}\left(t ; \hat{\theta}_{h 1}\right)\right)-U^{-}\left(c_{s s}^{-}(t)\right)-\hat{\theta}_{h 2} I(t) \\
& +\frac{\partial h}{\partial \theta_{h 1}}\left(t ; \hat{\theta}_{h}\right) \tilde{\theta}_{h 1}-I(t) \tilde{\theta}_{h 2}+O\left(\tilde{\theta}_{h}^{T} \tilde{\theta}_{h}\right)
\end{aligned}
$$

Truncate the higher order terms and re-arrange the previous expression into the matrix form

$$
e_{n l}=\tilde{\theta}_{h}^{T} \Phi
$$

where the nonlinear error term $e_{n l}$ depends on the parameter estimates $\hat{\theta}_{h}$

$$
\begin{aligned}
e_{n l}= & V(t)-\frac{R T}{\alpha F} \sinh ^{-1}\left[\frac{I(t)}{2 a^{+} A L^{+} i_{0}^{+}\left(c_{s s}^{+}\left(t ; \hat{\theta}_{h 1}\right)\right)}\right] \\
& +\frac{R T}{\alpha F} \sinh ^{-1}\left[\frac{I(t)}{2 a^{-} A L^{-} i_{0}^{-}\left(c_{s s}^{-}(t)\right)}\right] \\
& -U^{+}\left(c_{s s}^{+}\left(t ; \hat{\theta}_{h 1}\right)\right)+U^{-}\left(c_{s s}^{-}(t)\right)+\hat{\theta}_{h 2} I(t)
\end{aligned}
$$

and the regressor vector $\Phi$ is defined as:

$$
\Phi=\left[\frac{\partial h}{\partial \theta_{h 1}}\left(t ; \hat{\theta}_{h}\right),-I(t)\right]^{T}
$$

which depends upon measured signals and parameter estimates.
We now choose a least-squares parameter update law:

$$
\begin{aligned}
& \dot{\hat{\theta}}_{h}=P_{h} e_{n l} \Phi \\
& \dot{P}_{h}=-P_{h} \frac{\Phi \Phi^{T}}{m_{h}^{2}} P_{h}, \quad P_{h}(0)=P_{h 0}=P_{h 0}^{T}>0 \\
& m_{h}^{2}=1+\gamma_{h} \Phi^{T} \Phi, \quad \gamma_{h}>0
\end{aligned}
$$

\section{ADAPTIVE OUTPUT FUNCTION INVERSION}

In Section 3 we discussed how a linear state observer can be designed by using boundary values of the PDE. These boundary values must be processed from measurements by inverting the nonlinear output function. In this section we design an adaptive output function inversion scheme which utilizes the parameter estimate $\theta_{h}$ generated from Section 5 .

Our goal is to solve $g\left(c_{s s}^{-}, t\right)=0$ for $c_{s s}^{-}$, where

$$
\begin{aligned}
g\left(c_{s s}^{-}, t\right)= & \frac{R T}{\alpha F} \sinh ^{-1}\left[\frac{I(t)}{2 a^{+} A L^{+} i_{0}^{+}\left(c_{s s}^{+}\left(t ; \hat{\theta}_{h 1}\right)\right)}\right] \\
& -\frac{R T}{\alpha F} \sinh ^{-1}\left[\frac{I(t)}{2 a^{-} A L^{-} i_{0}^{-}\left(c_{s s}^{-}(t)\right)}\right] \\
& +U^{+}\left(c_{s s}^{+}\left(t ; \hat{\theta}_{h 1}\right)\right)-U^{-}\left(c_{s s}^{-}(t)\right)-\hat{\theta}_{h 2} I(t)-V(t)
\end{aligned}
$$

The main idea is to construct an ODE whose equilibrium satisfies $g\left(c_{s s}^{-}, t\right)=0$ and is locally exponentially stable. This can be viewed as a continuous-time version of Newton's method for solving nonlinear equations [21]. Consider the ODE

$$
\frac{d}{d t}\left[g\left(\check{c}_{s s}^{-}, t\right)\right]=-\gamma g\left(\check{c}_{s s}^{-}, t\right)
$$

whose equilibrium satisfies $g\left(c_{s s}^{-}, t\right)=0$. We expand and rearrange this equation into the familiar Newton's update law

$$
\frac{d}{d t} \check{c}_{s s}^{-}=-\left[\frac{\partial g}{\partial c_{s s}^{-}}\left(\check{c}_{s s}^{-}, t\right)\right]^{-1}\left[\gamma g\left(\check{c}_{s s}^{-}, t\right)+\frac{\partial g}{\partial t}\left(\check{c}_{s s}^{-}, t\right)\right]
$$

One can prove Lyapunov stability of this ODE, given appropriate bounds $\partial g / \partial c_{s s}^{-}$and $\partial g / \partial t$. The state $\check{c}_{s s}^{-}$of ODE (56) provides a recursive estimate of the surface concentration $c_{s s}(t)$ from measured current and voltage data, adapted according to the parameter estimate $\hat{\theta}_{h}$. The processed surface concentration $\check{c}_{s s}^{-}$supplies the "measured output" for the state estimator in Section 3.

Note that analytically proving stability of the fully-composed estimator is difficult. Consequently, we utilize simulation studies described in the following section.

\section{SIMULATIONS}

In this section we present numerical experimental results, which demonstrate the adaptive PDE observer's performance. 

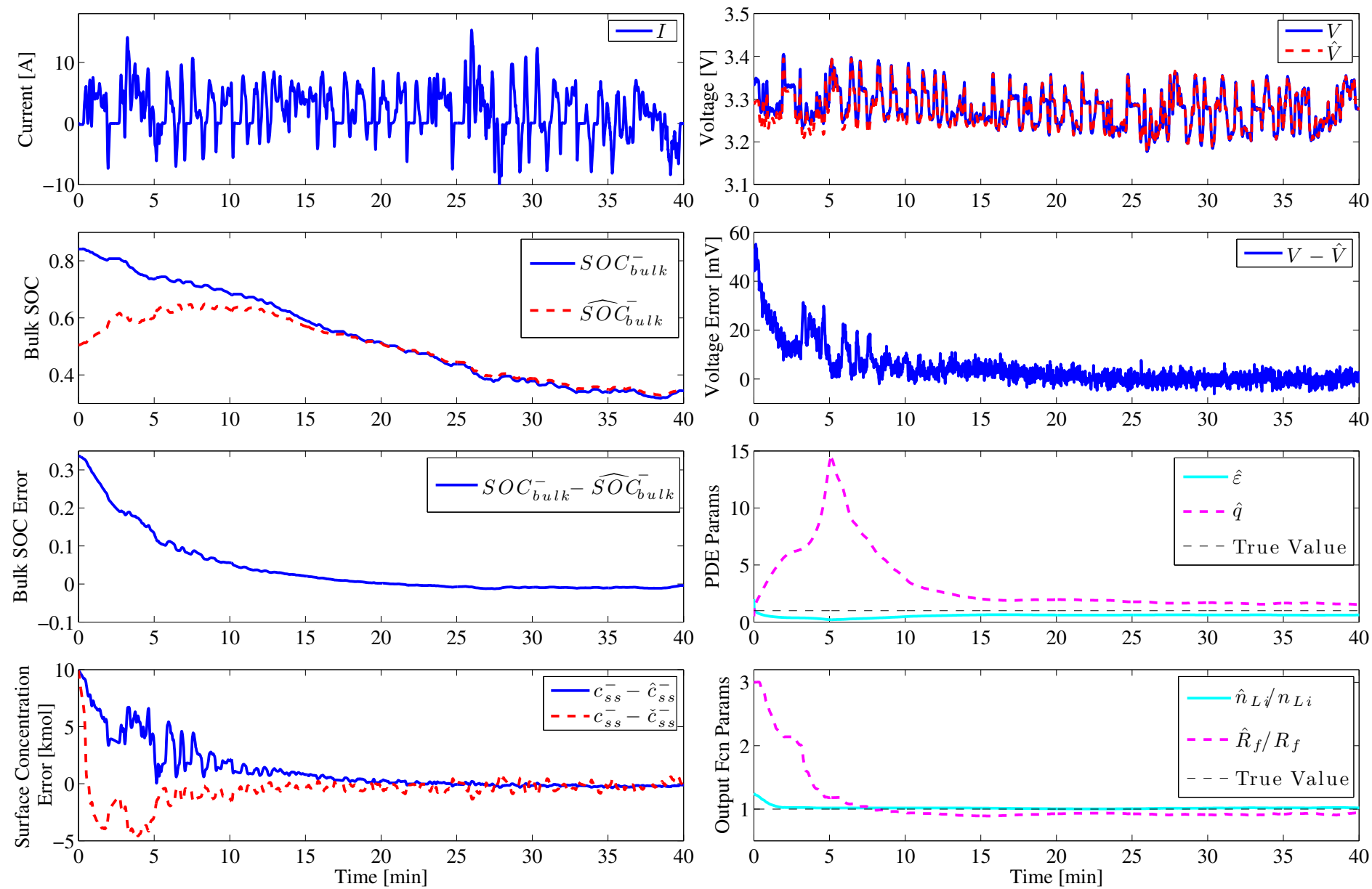

Figure 4. STATE AND PARAMETER ESTIMATION RESULTS FOR UDDSX2 DRIVE CYCLE. ZERO MEAN GAUSSIAN NOISE WITH A 2 mV VARIANCE WAS ADDED TO THE VOLTAGE MEASUREMENT. THE SPM PROVIDES THE "MEASURED" PLANT DATA. STATE AND PARAMETER ESTIMATES WERE INITIALIZED WITH INCORRECT VALUES.

Specifically, we apply the observer to the original SPM (includes diffusion in both electrodes). The model parameters used in this study originate from the genetic algorithm-based parameter identification study performed on commercial lithium-iron phosphate cells in [19]. For these parameters the characteristic diffusion times $\left(R_{s}^{2} / D_{s}\right)$ are $745 \mathrm{sec}$ and $0.32 \mathrm{sec}$ for the anode and cathode, respectively, which supports the argument for approximating cathode diffusion as instantaneous.

The state and parameter estimates are initialized at incorrect values: $\hat{c}_{s}^{-}(r, 0)=0.6 c_{s}^{-}(r, 0), \hat{\varepsilon}(0)=2, \hat{q}=0.5$, $\hat{n}_{L i}(0)=1.25 n_{L i}, \hat{R}_{f}(0)=3 R_{f}$. Moreover, zero mean normally distributed noise with a standard deviation of $2 \mathrm{mV}$ is added to the voltage measurement. Figure 4 portrays the evolution of the state and parameter estimates. The state estimates are represented by the bulk SOC, defined in (57), and surface concentrations. The surface concentration error includes the state estimation error $c_{s s}^{-}-\hat{c}_{s s}^{-}$and output function inversion error $c_{s s}^{-}-\check{c}_{s s}^{-}$. The PDE parameter estimates $\hat{\varepsilon}, \hat{q}$ and output function parameter estimates $\hat{n}_{L i}, \hat{R}_{f}$ also converge near their true values. The results are sim- ilar for various other initial conditions and drive cycle inputs, including US06, SC04, LA92, and naturalistic micro trip data.

$$
\operatorname{SOC}_{b u l k}^{-}(t)=\frac{3}{c_{s, \max }^{-}} \int_{0}^{1} r^{2} c_{s}^{-}(r, t) d r
$$

\section{CONCLUSION}

This paper reports on the first combined SOC/SOH estimator for electrochemical battery models. The adaptive observer utilizes concepts from PDE estimation and adaptive control theory to generate various new concepts for battery systems and control. These are summarized by four key ideas: First, a backstepping PDE state estimator is designed in previous work [20]. Second, a Padé approximation of the transfer function for lithium diffusion is used to identify the diffusion coefficient. Third, parameter sensitivity analysis is applied to elucidate the linear dependence between physically meaningful parameters related to capacity and power fade. Fourth, an adaptive output function inversion tech- 
nique enables linear state estimation designs. Finally, we present simulations which demonstrate how the adaptive observer successfully identifies the states and parameters from measurements of voltage and current, only. The composition of these unique ideas provides a combined $\mathrm{SOC} / \mathrm{SOH}$ estimation algorithm for battery systems using electrochemical models.

Our next steps include state/parameter estimation schemes for the complete Doyle-Fuller-Newman model [5]. This work will enable output feedback controllers for optimal charging and discharging.

\section{REFERENCES}

[1] Chaturvedi, N. A., Klein, R., Christensen, J., Ahmed, J., and Kojic, A., 2010. "Algorithms for advanced batterymanagement systems". IEEE Control Systems Magazine, 30(3), pp. $49-68$.

[2] Moura, S., Fathy, H., Callaway, D., and Stein, J., 2011. "A Stochastic Optimal Control Approach for Power Management in Plug-In Hybrid Electric Vehicles". IEEE Transactions on Control Systems Technology, 19(3), pp. 545 -555.

[3] Siegel, J. B., Lin, X., Stefanopoulou, A. G., Hussey, D. S., Jacobson, D. L., and Gorsich, D., 2011. "Neutron imaging of lithium concentration in LFP Pouch cell battery". Journal of the Electrochemical Society, 158(5), pp. A523 - A529.

[4] Liu, P., Wang, J., Hicks-Garner, J., Sherman, E., Soukiazian, S., Verbrugge, M., Tataria, H., Musser, J., and Finamore, P., 2010. "Aging Mechanisms of LiFePO4 Batteries Deduced by Electrochemical and Structural Analyses". Journal of the Electrochemical Society, 157(4), pp. A499-A507.

[5] Thomas, K., Newman, J., and Darling, R., 2002. Advances in Lithium-Ion Batteries. Kluwer Academic/Plenum Publishers, New York, NY USA, ch. 12: Mathematical modeling of lithium batteries, pp. 345-392.

[6] Plett, G. L., 2004. "Extended Kalman filtering for battery management systems of LiPB-based HEV battery packs. Part 3. State and parameter estimation". Journal of Power Sources, 134(2), pp. 277-92.

[7] Verbrugge, M., and Tate, E., 2004. "Adaptive state of charge algorithm for nickel metal hydride batteries including hysteresis phenomena". Journal of Power Sources, 126(1-2), pp. 236-249.

[8] Verbrugge, M., 2007. "Adaptive, multi-parameter battery state estimator with optimized time-weighting factors". Journal of Applied Electrochemistry, 37(5), pp. 605 - 616.

[9] Hu, Y., and Yurkovich, S., 2012. "Battery cell state-ofcharge estimation using linear parameter varying system techniques". Journal of Power Sources, 198, pp. 338 - 350.
[10] Santhanagopalan, S., and White, R. E., 2006. "Online estimation of the state of charge of a lithium ion cell". Journal of Power Sources, 161(2), pp. 1346 - 1355.

[11] Domenico, D. D., Stefanopoulou, A., and Fiengo, G., 2010. "Lithium-Ion Battery State of Charge and Critical Surface Charge Estimation Using an Electrochemical Model-Based Extended Kalman Filter". Journal of Dynamic Systems, Measurement, and Control, 132(6), p. 061302.

[12] Smith, K. A., Rahn, C. D., and Wang, C.-Y., 2008. "Modelbased electrochemical estimation of lithium-ion batteries". In 2008 IEEE International Conference on Control Applications, pp. 714-19.

[13] Klein, R., Chaturvedi, N. A., Christensen, J., Ahmed, J., Findeisen, R., and Kojic, A., 2012. "Electrochemical Model Based Observer Design for a Lithium-Ion Battery". IEEE Transactions on Control Systems Technology, PP, pp. 1-13.

[14] Santhanagopalan, S., Guo, Q., Ramadass, P., and White, R. E., 2006. "Review of models for predicting the cycling performance of lithium ion batteries". Journal of Power Sources, 156(2), pp. 620-628.

[15] Chen, C., 1998. Linear System Theory and Design. Oxford University Press, Inc.

[16] Krstic, M., and Smyshlyaev, A., 2008. Boundary Control of PDEs: A Course on Backstepping Designs. Society for Industrial and Applied Mathematics, Philadelphia, PA.

[17] Delacourt, C., Poizot, P., Levasseur, S., and Masquelier, C., 2006. "Size effects on carbon-free LiFePO4 powders". Electrochemical and Solid-State Letters, 9(7), p. A352.

[18] Derrien, G., Hassoun, J., Panero, S., and Scrosati, B., 2007. "Nanostructured $\mathrm{Sn}-\mathrm{C}$ composite as an advanced anode material in high-performance lithium-ion batteries". Advanced Materials, 19(17), p. 2336.

[19] Forman, J. C., Moura, S. J., Stein, J. L., and Fathy, H. K., 2012. "Genetic identification and Fisher identifiability analysis of the Doyle-Fuller-Newman model from experimental cycling of a LiFePO4 cells". Journal of Power, 210, pp. 263275.

[20] Moura, S. J., Chaturvedi, N., and Krstic, M., 2012. "PDE Estimation Techniques for Advanced Battery Management Systems - Part I: SOC Estimation". In Proceedings of the 2012 American Control Conference.

[21] Ioannou, P., and Sun, J., 1996. Robust Adaptive Control. Prentice-Hall.

[22] Smyshlyaev, A., and Krstic, M., 2010. Adaptive Control of Parabolic PDEs. Princeton University Press.

[23] Lund, B. F., and Foss, B. A., 2008. "Parameter ranking by orthogonalization - Applied to nonlinear mechanistic models". Automatica, 44(1), pp. $278-281$. 